\title{
Firearm Detection Using Wrist Worn Tri-Axis Accelerometer Signals
}

\author{
Md Abdullah Al Hafiz Khan*, David Welsh ${ }^{\dagger}$, and Nirmalya Roy ${ }^{\ddagger}$ \\ Department of Information Systems \\ University of Maryland, Baltimore County \\ Email: *mdkhan1@umbc.edu, ${ }^{\dagger}$ dwelsh2@umbc.edu, ${ }^{\ddagger}$ nroy@umbc.edu,
}

\begin{abstract}
Gunshot detection traditionally has been a task performed with acoustic signal processing. While this type of detection can give cities, civil services and training institutes a method to identify specific locations of gunshots, the nature of acoustic detection may not provide the fine-grained detection accuracy and sufficient metrics for performance assessment. If however you examine a different signature of a gunshot, the recoil, detection of the same event with accelerometers can provide you with persona and firearm model level detection abilities. The functionality of accelerometer sensors in wrist worn devices have increased significantly in recent time. From fitness trackers to smart watches, accelerometers have been put to use in various activity recognition and detection applications. In this paper, we design an approach that is able to account for the variations in firearm generated recoil, as recorded by a wrist worn accelerometer, and helps categorize the impulse forces. Our experiments show that not only can wrist worn accelerometers detect the differences in handgun rifle and shotgun gunshots, but the individual models of firearms can be distinguished from each other. The application of this framework could be extended in the future to include real time detection embedded in smart devices to assist in firearms training and also help in crime detection and prosecution.
\end{abstract}

\section{INTRODUCTION}

How participants of shooting sports are able to transfer recoil impulses generated from the discharge of firearms to the ground can affect accuracy, general safety, and overall endurance. As outlined in the governing Test Operation Procedure 3-2-504 set forth in 1977, the equation for recoil energy and limits for daily firing can be used as a means to compare and group calculated recoil with recorded accelerometer sensor data. Using this equation as a starting point, we will examine how firearms can take generated recoil, and impart it into the user in varying ways. The interaction between user and weapon plays an important role in recoil mitigation, along with the different mitigation techniques implemented in firearms [1]. As examined in [2] the recoil energy generated from a firearm may differ from the perceived recoil.

Mitigation techniques that are implemented into the design of firearms can greatly reduce recoil and are not taken into account with this basic recoil equation. This can include action/ auto loading features, muzzle brakes and recoil pads among others. Other main influences on recoil are differences in the basic types of firearms. The types being examined in this study are pistols, rifles and shotguns. For handguns, the only point of contact between the user and the firearm is near the wrist. This rearwards generated recoil is imparted into the user differently from that of rifles and shotguns. With rifles and shotguns, a user typically has 3 points of contact with the firearm: shoulder, dominant hand at the trigger and nondominant hand on a forward handguard/ grip. These contact points, depending on the users grip and design of the firearm regardless of type are examples of how recoil can be passed through a user.

Taking into consideration these differences in firearms and how a typical user handles and fires them in a safe manner, we examine how wrist worn accelerometers could detect these basic differences. Typical detection research of firearms and gunshots have been acoustical in nature [3]. Acoustic features generated by a firearm have also been examined along with performance of detection algorithms [4]. This can be beneficial in detection systems employed within cities, however one variable within a firearm such as barrel length can influence acoustical features of a gunshot [5]. To better address the high variability seen within basic firearm design and usage, we examine how 15 different firearms of similar and differing calibers create similar and dissimilar patterns in sensor readings from a wrist worn accelerometer. Potential applications include crime evidence collection where everyday accelerometer sensors such as those in fitness sensors and smart watches record gunshot recoil. In regards to gunshots, acoustic detection could identify an area and direction of a gunshot. Gunshot recoil sensor data will be able to identify the person who held the firearm since recoil is contact specific and not easily transferred between people. This level of detection could prove useful to law enforcement as unobtrusive and pervasive sensor usage increases. Perpretrators of violent crimes could be identified if sensors worn during crimes recorded gunshot signatures. Data could be analyzed to identifying signatures of shooter specific recoil. Military and law enforcement may also benefit from the application of recoil detection, characterization, and classification during training. Differences in firearms and users can be analyzed easier based on the actual felt recoil and not based off a simple calculation.

Main contribution of this paper is as follows.

- We study the problem of various types of firearm detection in this experiment. Three categories of firearms \{"handgun', 'rifle', 'shotgun'\} are used in this work. To infer individual firearm, we build three classifier model with Decision Tree (DT) classifier for each of the 
categories. We use SVM based approach to recognize whether a firearm is autoloaded or manual.

- Firearm shooting generates recoil force and impacts the sensor signals. To collect firearm shooting accelerometer signal with the help dominant hand worn accelerometer. In this work, fifteen different firearms are deployed for collecting firearm shooting dataset.

- We evaluate the efficacy of our proposed framework with the collected fifteen firearm shooting dataset. We demonstrate how our proposed framework helps determine categories of firearm, firearm and the type of the firearm.

\section{RELATED WORK}

By examining how recoil is transferred to a shooter through the use of accelerometers, the differences in firearms can be examined. This was examined briefly in [6] where recoil force was compared between different weapons but with sensors embedded into the rifle and not the user. [7] used wrist worn accelerometers in order to develop a recoil based gunshot detection algorithm where the signature of the recoil generated by a handgun was examined. A later study examined the same principles of gunshot detection but with the use of a smartphone [8] where user behavior and sensor fusion was utilized. The effects of recoil was examined in [9] but focused on its impact on accuracy. Their research indicated that recoil did not influence accuracy of the shot but the subjects shooting position, experience and movement leading up to the shot had the greatest impact on accuracy.

One limitation to these studies is that they do not examine the differences in firearms and the associated recoil or attempt to account for the differences in how users may mitigate the recoil. This issue had been researched in the "British Textbook of Small Arms" published in 1929 and again in the SAAMI Technical Data Sheet Technical Correspondent's Handbook of 1976. Both of these still do not fully take into account the wide variations of barrel length and other firearm recoil mitigation techniques.

The effects of barrel length on bullet velocity was examined in [5]. Direct port pressure measurement was conducted with a piezoelectric transducer, while velocity (at 2 meters), and sound pressure level were recorded for each shot. Their study showed how changing one variable in a firearm (barrel length) could change the behavior of a firearm. Pressure in the bore (uncorking pressure) and sound pressure level increased exponentially as barrel length decreased and velocity decreased from $2,964 \mathrm{fps}$ at $24^{\prime \prime}$ to $1,823 \mathrm{fps}$ at $5^{\prime \prime}$. Current detection and classification methods have not covered this variation in firearm performance within their studies.

The ShotMaxx timer developed by Double Alpha-Academy [10] utilizes sound and accelerometers for gunshot detection. Shot timers do not take into account the type of firearm since the purpose is to detect and track times between shots for training and competitive shooting. When examining gunshot detection algorithms in [4], signal preprocessing focused on acoustics of firearms not on recoils. Even within the area of activity recognition, one of the main differences with

\begin{tabular}{|c|c|c|c|}
\hline Category & Firearm & Type (Autoloading) & Caliber \\
\hline \multirow{3}{*}{ Handgun } & Ruger MK2 & Yes & $22 \mathrm{LR}$ \\
& FN 5.7 & Yes & $5.7 \times 28$ \\
& Glock 19 & Yes & $9 \mathrm{~mm}$ \\
\hline \multirow{5}{*}{ Rifle } & Ruger 10/22 & Yes & $22 \mathrm{LR}$ \\
& Bolt Action .22 & No & $22 \mathrm{LR}$ \\
& ADCOR AR15 & Yes & $5.56 \times 45$ \\
& M16 AR15 & Yes & $5.56 \times 45$ \\
& Yugo SKS & No & $7.62 \times 39$ \\
& WASR 10 AK47 & Yes & $7.62 \times 39$ \\
& Scoped M91/30 & No & $7.62 \times 54 \mathrm{R}$ \\
& Romanian PSL & Yes & $7.62 \times 54 \mathrm{R}$ \\
& M44 & No & $7.62 \times 54 \mathrm{R}$ \\
\hline \multirow{5}{*}{ Shotgun } & Remington 1100 & Yes & 12 Gauge \\
& VEPR 12 & Yes & 12 Gauge \\
& Remington 870 & No & 12 Gauge \\
\hline
\end{tabular}

TABLE I: The table represents the set of firearms, their corresponding category, autoloading types and caliber.

gunshot detection and other typical activities is the duration of the event. Muzzle blasts last for around 5 milliseconds as examined in [4], and recoil evens were found to last for around 50 milliseconds for handguns in [7]. The sampling rate for this study was reduced to $1,600 \mathrm{~Hz}$ but it is still much higher than rates used for activity recognition. For this application the sampling rate allows for better collection of the short lived events created by gunshot recoil, aiding in feature extraction. A sampling rate of $100 \mathrm{~Hz}$ was applied to a real time pothole detection application in [11]. Their approached applied different methods including thresholds examined with sliding window, consecutive measurements, and basic values above and below certain levels all designed to run on Android smartphones. Another smartphone based study examined the performance of hotword detection utilizing accelerometers [12]. One of the limitations on audio signal processing is the collection rate required and the subsequent computational power needed to process it whether it is for gunshot detection or activity recognition. Their solution instead utilized an accelerometer at $200 \mathrm{~Hz}$ with a high-pass filter to remove interference can reduce energy efficiency while retaining an accuracy of $98 \%$. Both of these methods required the processing of accelerometer data for quick short lived events such as potholes detected while driving, or recognizing keywords spoken by a user.

\section{System Design AND Algorithm}

In this work, we focus on recognizing firearm described in Table I. Raw accelerometer sensor signals from gunshots of each firearm is processed to examine the firearm and it's type. We remove noise and generate frames from the signal. Gunshot produces recoil for a short amount of time hence not all the frames contains gunshot related signals and need to be discarded. We admit only the gunshot related signals and determine the specific firearm and type. Figure 1 represents our proposed firearm detection framework. Our framework comprises of two component $i$ ) Data Processing and ii) Recognition Modeling. Data processing component processes raw accelerometer signals and generate features while the recognition modeling use these extracted features to infer specific firearm. We discuss the details of our framework in the next section. 


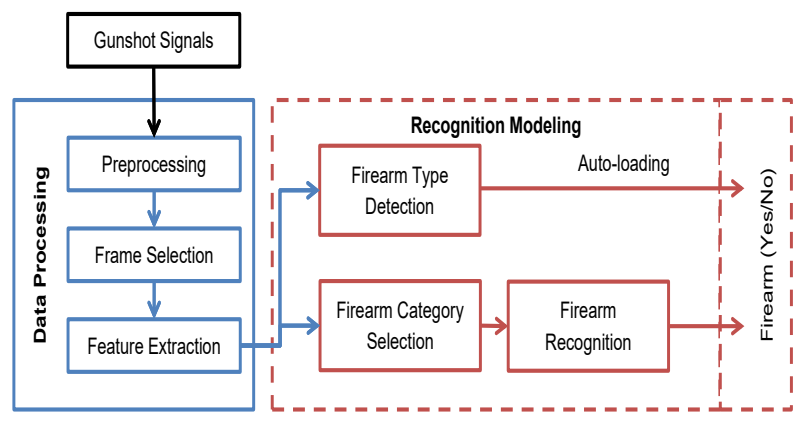

Fig. 1: Firearm Detection Framework

\section{A. Data Processing}

This module comprises of $i$ ) Preprocessing, ii) Frame Selection and iii) Feature Extraction. Preprocessing filters the raw accelerometer signals and create frames. Frame selection admits gunshot related frames to the feature extractor which extracts statistical and frequency domain features. we discuss the details as below.

i) Preprocessing: While collecting the firearm data, we ensure datasets contains only successful gunshots by removing non-gunshot related signals such as removing jams or the rounds that failed the fire. Typical auto loading handguns rifles or shotguns do not require the user to load a new round after the trigger is pulled, they simply release the trigger and are ready to fire the next round. However, non-auto loading firearms require the user to cycle a new round. One shotgun in this study was pump action, and four rifles were bolt action. While these features are not directly related to gunshots, they are retained in the datasets. Once we collect the gunshot dataset, we deploy low-pass filter to remove noise. Upon examining the frequency range of different types of guns, we determine the cutoff frequency to $150 \mathrm{~Hz}$. Frames are created from these filtered accelerometer signals. We create frames from the accelerometer signals using sliding window based approach with 50\% overlap. Each window contains 1600 sample data points. These frames are then fed into the frame selection module for further processing.

i) Frame Selection: In this module, we admit only the gunshot related frames and discards all unrelated frames to further get the fine-grained gunshot frames. Accelerometer signals show typical short $(10 \mathrm{~ms})$ high peak values due to recoil force of the gun. Most of the non spike signal remains flat and do not contribute to the firearm detection process. We discard the frames that do not contain firearm related information. For this purpose, we calculate energy of the frames, $E=\sum_{i=0}^{L}\left(x^{2}+y^{2}+z^{2}\right)$, where $L$ is the window length in terms of the number of samples. Once the energy of the frame $E$, crosses the threshold $\delta_{e}$ (determined with empirical evaluation), we select this frame for the feature extraction process and discard frames that fails to meet the threshold. Figure 2 represents frame selection strategy of our framework.

i) Feature Extraction: We extracted relevant time domain and frequency domain features from the selected frames. Gunshot related frames have high spikes in the signals and

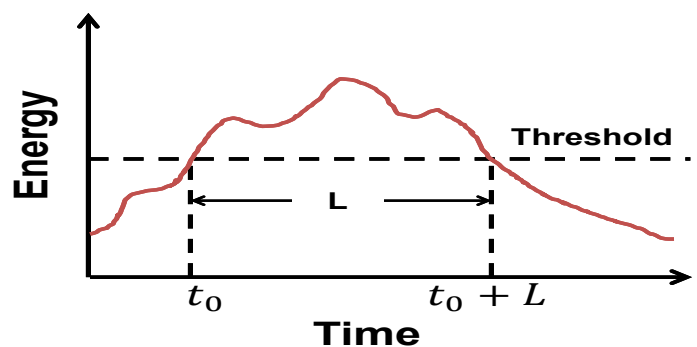

Fig. 2: Frame Selection Approach. L represents frame length. Threshold is shown with horizontal dashed line.

therefore time domain features play a significant role in firearm determination. Time domain features like mean, standard deviation, signal magnitude area, energy are calculated. We employ Hamming window to normalize the frequency and compute Fast Fourier Transform (FFT) on each frame. We then compute frequency domain energy for each frame.

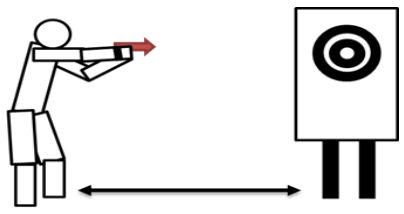

(a)

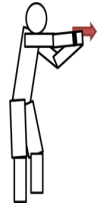

(b)

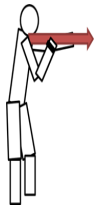

(c)
Fig. 3: Shooting setting and styles. (a) Shooting the target with handgun, (b) Handgun shooting style, and (c) Rifle/Shotgun shooting style

\section{B. Recognition Modeling}

This module consists of $i$ ) Firearm Type Detection, ii) Category Selection and iii) Firearm Recognition. Firearm type detection infers whether a firearm is autoloaded or not. Category selection determines in which category a firearms belongs to (i.e., handgun, rifle, shotgun). Firearm classification infers individual firearms depending on the category. The details are discussed below.

i) Firearm Type Detection: Given the set of features, our task is to train a firearm type detector in order to infer whether a firearm is autoloaded or manual. Of the 15 firearms, 10 are autoloading. This provides a larger dataset using autoloaded gunshot samples as positive samples for training, and manual firearms for negative. A one-class Support Vector Machine
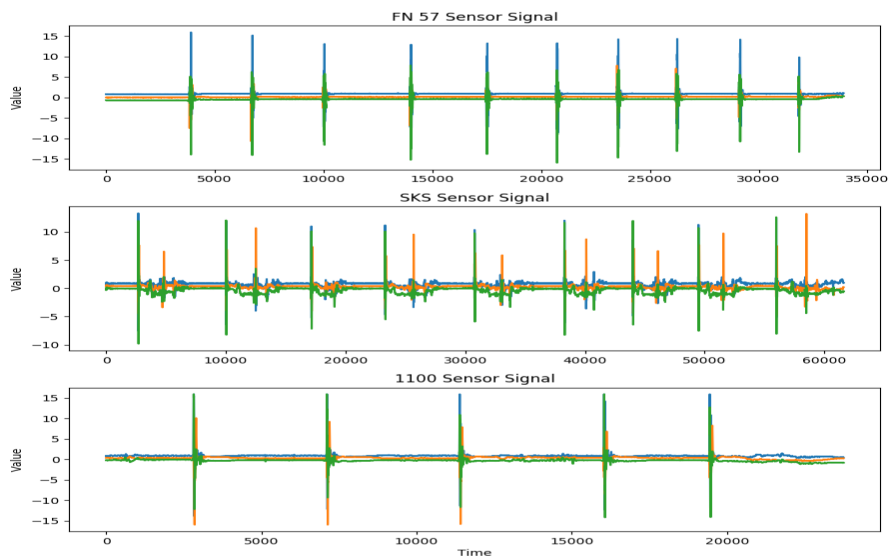

Fig. 4: Accelerometer sensor signals of different gunshot 


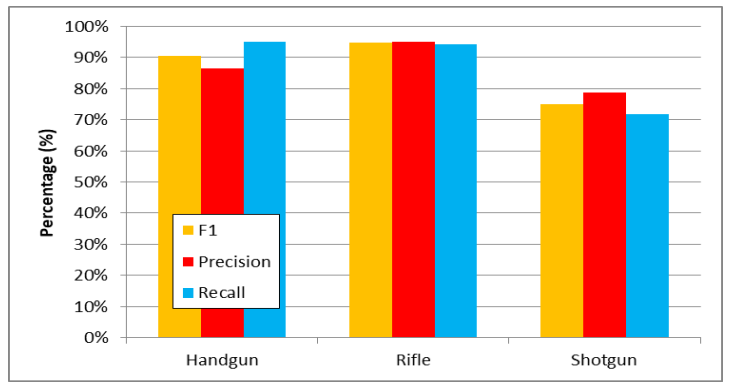

Fig. 5: Firearm Category Detection Performance

(SVM) classifier [13] is trained with only the autoloaded gunshot samples as positive instances. In the testing phase both positive and negative samples are passed through this trained model to determine whether samples come from autoloaded gun or manual gun.

ii) Category Selection: In this phase, we determine which category of firearm a gunshot comes from \{'handgun', 'rifle', 'shotgun'\}. Our training class contains the firearms from all three classes. We then train a Decision Tree (DT) classifier [14] with the annotated samples of the three classes. We use DT for the simplicity and ease of implementation and ability to perform realtime detection. In the testing phase, samples are passed through this constructed tree to determine the final class labels (gun type).

iii) Firearm Recognition: In this work, firearm classification is performed by employing a DT [14]. This classifier builds a tree structure using annotated features from the training data and each leaf represents class labels. In the testing phase, this constructed tree is traverses through the branch to determine the firearm classifier. The DT can be implemented with simple if else conditions, hence lower computation complexity. In this experiment, we construct a DT for each category and we use corresponding gunshot data to train the DT classifier. In the testing phase, once a category is determined the corresponding DT model is used to infer the firearm.

\section{Experimental Setup and Evaluation}

\section{A. Data Collection}

In this experiment, an AX3 Watch, Axivity Ltd triaxis accelerometer sensor is used. Data collection was performed at $1600 \mathrm{~Hz}$ with offset $\pm 16 \mathrm{~g}$ based on performance during the calibration tests. To ensure the safety of participants only one user performed the shooting for this initial study. The age of the participant was 27 years and height and weight was 6 '2" and 1801 lbs respectively.

To reduce collection time all firearms where set up beforehand and loaded with the pre-determined number of rounds of ammo. This reduced the number of actions the shooter would need to take to fire the weapon, eliminating non gunshot noise from the data such as loading magazines and releasing firearm bolts and slides. With the accelerometer recording the subject was then handed firearms in order of lowest calculated recoil to highest, handguns followed by rifles and shotguns. Due to safety concerns, firearms with calculated recoil under $10 \mathrm{ft}$. lbs. were loaded with 10 rounds while firearms with calculated

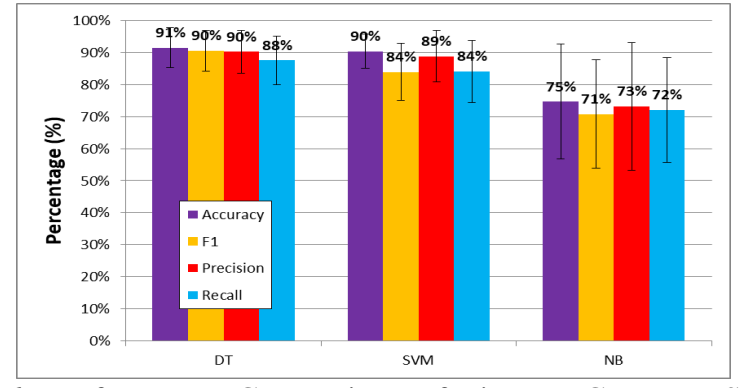

Fig. 6: Performance Comparison of Firearm Category Selection

recoils over 10 were only loaded with 5 rounds. The subject was instructed to take aimed deliberate shots like they would for target practice. This was done at a private shooting range on flat level ground with a target placed at torso height 10 yards down range. All shots were performed with the shooter in the standing position; feet shoulder width apart and shooting all firearms unsupported. After firing all rounds for each firearm, the next firearm was handed to the shooter. During firing the subject was instructed to clear any jams or malfunctions normally and these incidents were noted for data processing. Start and stop times were recorded to ensure accurate data processing. The shooting setup, difference in weapon grips, and accelerometer sensor signals are shown in Figure 3 and Figure 4.

\section{B. Performance Metrics}

We evaluated our framework based on standard precision, recall, f1 score and accuracy. We also compare our framework performance with standard classifier Naive Bayes (NB) [15], Support Vector Machine (SVM) [16] classifier.

\section{Experimental Results}

We evaluated our firearm detection framework with fifteen firearms' gunshot data traces and demonstrated individual component performances. We also discussed overall performance of our framework as well.

i) Category Inference Accuracy: Selected feature frames are fed into the firearm category selection module to infer the firearm categories. In this experiment, different firearm gunshot data is aggregated based on the firearm category to create category dataset containing three classes - 'handgun', 'rifle', 'shotgun'. We then randomly split the dataset into training and testing dataset. Our training dataset contains approximately $70 \%$ data and testing dataset contains $30 \%$ data. We train our DT classifier with the training dataset and evaluate the trained model with testing dataset. Figure 5 represents category selection performance of our framework. We see that our framework achieves F1 scores approximately 90\%, 93\% and $76 \%$ for handgun, rifle and shotgun respectively. We observe that handgun and rifle gunshot signals are more distinctive compare to shotgun, hence it achieves lower F1 score.

ii) Firearm category selection performance comparison: We also compare our firearm category selection performance against SVM and NB classifier. In this experiment, we use 


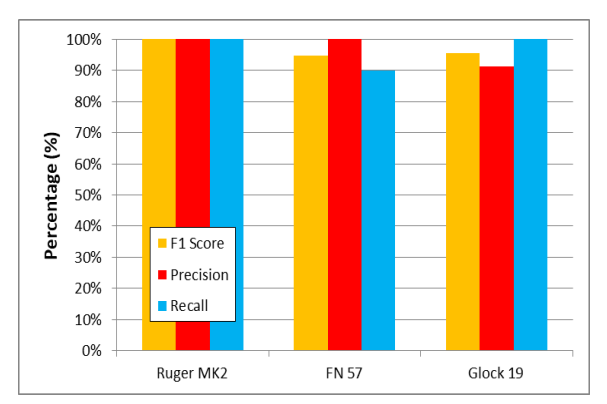

(a)

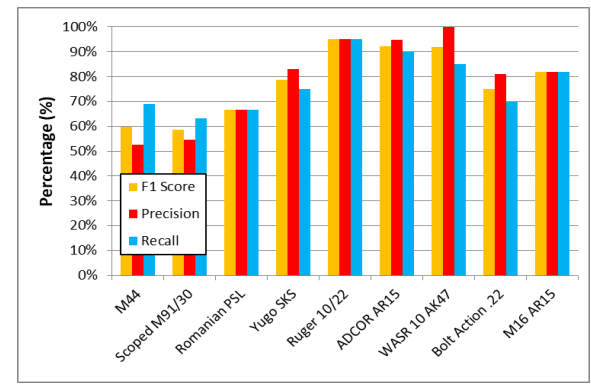

(b)

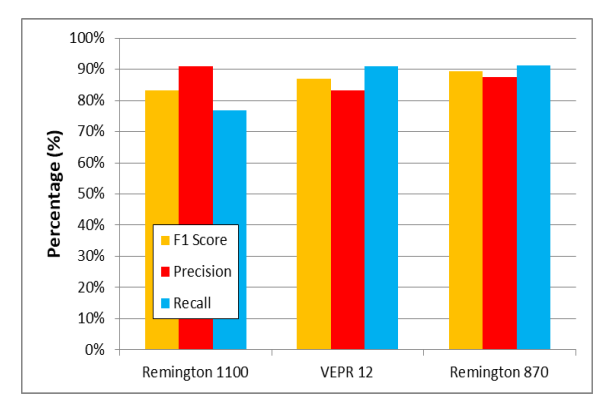

(c)

Fig. 7: Firearm detection performance of our framework. (a) Handgun detection performance, (b) Rifle detection performance, and (c) Shotgun detection performance

standard 10-fold cross-validation technique to evaluate our framework performance. Figure 6 represents the performance comparison of our firearm category selection performance. From the figure, we see that our framework performance gain approximately $17 \%$ compare to NB classifier. Our DT based approach performs better because it ables to partition the feature space into three category classes more appropriately than the NB classifier.

iii) Firearm Type Detection Performance: In this experiment, we evaluate the performance of our firearm type detector performance. We use one-class SVM and determine whether a firearm is autoloaded or manual. We trained two SVM model. First SVM model is trained with autoloaded firearm samples from all the gunshot as positive classes and second SVM model with manual firearm instances as positive classes. The training dataset for each classes was splitted randomly to generate the training and validation dataset. We use the testing samples to measure the performance of these two SVM model performance. The average of these results are presented in Table II. We see that our model achieves $\approx 90 \%$ accuracy on average. Our firearm type detector capable detecting whether a firearm is autoloaded or manually $90 \%$ times correctly.

\begin{tabular}{|c|c|c|c|}
\hline Accuracy (\%) & F1 score (\%) & Precision (\%) & Recall(\%) \\
\hline 90.40 & 94.96 & 1.0 & 90.40 \\
\hline
\end{tabular}

TABLE II: Firearm type detection performance (Percentage)

iv) Firearm Recognition Performance: We classify firearms in three separate categories. Therefore, we design three DT models to infer individual firearm class. We trained our DT model with the corresponding categories ('handgun', 'rifle', 'shotgun') firearms instances. Each categorys datasets are also randomly split into $70 \%$ training and 30\% testing data. DT models are trained with this $70 \%$ training data and evaluated with the remaining gunshot data. The detail results are reported in figure 7. Our handgun, rifle and shotgun have three, nine and three firearms respectively. From Figure $7 \mathrm{a}$ we observe that 'Ruger MK2' handgun detection performance is $100 \%$ as the recoil force of this firearm generates unique signals that helps detects all testing instances correctly. Utilizing the recoil equation provided in TOP-3-2-504 we calculated the recoil to be about 0.23 foot pounds. With the 'FN 57' and 'Glock 19', some samples are incorrectly classified. The calculated recoil was 2.29 and $4.45 \mathrm{ft}$ lbs respectfully and could indicate that recoil of similarly performing handgun could produce similar signals when compared against the MK2. Figure $7 \mathrm{~b}$ represents the individual rifles detection performance. We observed that 'M44' and 'Scoped M91/30' have F1 scores $60 \%$ and 58\% respectively as these firearms gunshot recoil have similar impact on accelerometer signal compared to other firearms. Note that these two both fired the same cartridge and are not auto-loading. We can also infer that frame selection module also admits more non-gunshot/ manual loading (in case of 'M44', 'Scoped M91/30') related frames and our firearm recognition model incorrectly classify these instances. From the figure, we also see that 'Ruger 10/22' achieves F1 score $93 \%$ as it has a unique low recoil force that is auto loading like the MK2 which fires the same cartridge. Figure $7 \mathrm{c}$ represents the shotgun detection performance. F1 scores for shotguns are $83 \%, 87 \%$ and $89 \%$, respectively. The similar performance for shotguns could be due to their high recoil maxing out the accelerometer signal resulting in similar recoil peaks when their calculated recoil actually ranges from about 20-34 ft lbs. We conclude that firearms with calculated recoil forces distinct (outliers) from other firearms generate more distinguishable accelerometer signals. Classes with fewer firearms like handguns and shotguns had less variation within their class in regards to caliber/ gauge and calculated recoil resulting in lower performance.

v) Firearm Recognition Performance Comparison: In this experiment, we compare the average performance of our individual firearm detection DT model. We perform 10-fold cross validation and keep track of the results. Figure 8 represents the average firearm detection performance for each individual firearm detection DT model. From Figure 8a, we see that average F1 scores for individual handgun detection are 93.77, 93.96 and 94.22 (percent) for DT, SVM and NB model, respectively. Our handgun DT model performs similar as SVM and NB model. In Figure 8b, average F1 scores of individual rifle firearm detection is $73.62 \%, 65.08 \%$ and $69.52 \%$ for DT, SVM and NB models. It is noted that with a higher number of individual rifle classes, SVM and NB model performance degrades compared to DT model. In Figure 8c, we note that average $\mathrm{F} 1$ scores of individual shotgun detection models are $88.41,77.97 \%$ and $82.41 \%$ respectively for DT, SVM and NB. We also observe that our DT model performance gain is $\approx 5$ - 


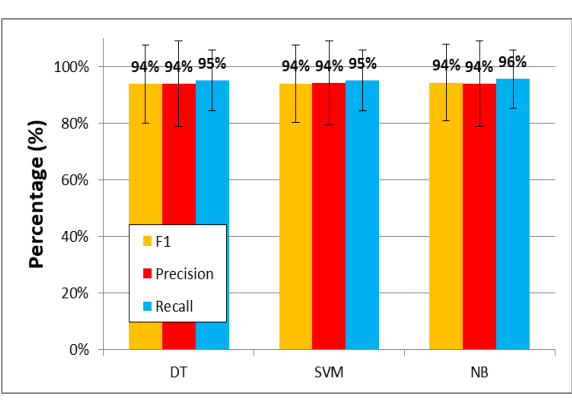

(a)

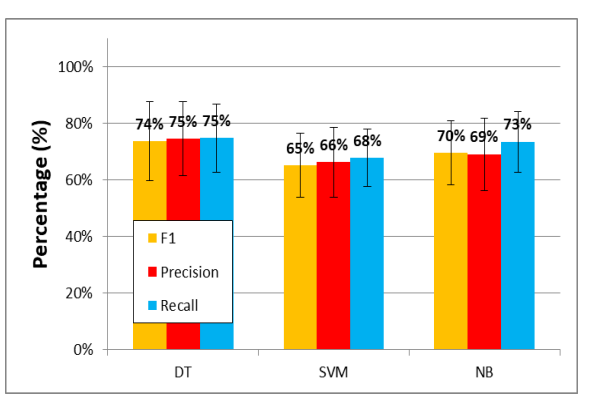

(b)

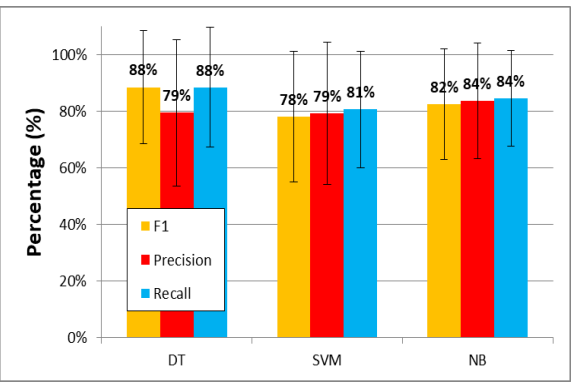

(c)

Fig. 8: Firearm detection performance comparison of our framework. (a) Handgun detection performance comparison, (b) Rifle detection performance comparison, and (c) Shotgun detection performance

$6 \%$ for individual handgun detection. We conclude that our individual firearm detection DT model performs better than other classifier when large number of different firearms shots present in the dataset.

\section{Discussion AND Future Work}

Limitations of this work include the volume of data analyzed. Safety and training requirements for a study of this nature restricted the number of gunshots and participants. Our model can be expanded to a multi user multi firearm study examining performance between users. Under similar conditions in [8], handgun recoil and gunshot sound was detected by smartphone sensors with sampling rates of under $200 \mathrm{~Hz}$. Examining the effects of sampling rate and system accuracy could provide a balanced and realistic approach that can be utilized in current wearables. The impact of different users can also be examined in future applications of this system. The interaction with a firearm could be examined by identifying differences in the recoil signals of the same firearm in different people. This system can be employed for multiple users to examine how their interactions impact accuracy and overall handling of the firearm.

\section{CONCLUSION}

Firearm shooting generates recoil force and this recoil force has an impact on the sensor signal. The recoil force is fairly unique for each firearm. We deployed a wrist worn accelerometer on the users dominant hand and collected firearm shooting signals of 15 different firearms shooting. These collected signals were then preprocessed to remove unwanted portion of the signal. We exploited the firearm shooting signals characteristic and select only firearm related frames for further processing. Our feature extractor module generates related feature sets for next step. In this work, we built DT based firearm category model and recognized \{'handgun', 'rifle', and 'shotgun'\}. We then built individual firearm recognition model for each of the categories using DT classifier. Our individual firearm detector achieves recognition accuracy $\approx 86.67 \%$. Finally, we used one-class SVM to determine the whether a firearm is autoloaded or manual type.

\section{ACKNOWLEDGMENT}

This research is partially supported by the ONR under grant N00014-15-1-2229.

\section{REFERENCES}

[1] Bruce P Burns. Recoil considerations for shoulder-fired weapons. Technical report, ARMY RESEARCH LAB ABERDEEN PROVING GROUND MD WEAPONS AND MATERIALS RESEARCH DIRECTORATE, 2012.

[2] Frank Morelli, Jennifer M Neugebauer, Michael E LaFiandra, Patricia Burcham, and Caleb T Gordon. Recoil measurement, mitigation techniques, and effects on small arms weapon design and marksmanship performance. IEEE Transactions on Human-Machine Systems, 44(3):422$428,2014$.

[3] Robert C Maher. Acoustical characterization of gunshots. In Signal Processing Applications for Public Security and Forensics, 2007. SAFE'07. IEEE Workshop on, pages 1-5. IEEE, 2007.

[4] Alfonso Chacon-Rodriguez, Pedro Julian, Liliana Castro, Pablo Alvarado, and Néstor Hernández. Evaluation of gunshot detection algorithms. IEEE Transactions on Circuits and Systems I: Regular Papers, 58(2):363-373, 2011.

[5] Dr. Philip H. Dater and Jason Wong. Barrel length studies in $5.56 \mathrm{~mm}$ nato weapons. Small Arms Defense Journal, 2012.

[6] D. P. Armstrong. The $20 \mathrm{~mm}$ anti-material rifle: A new use for unused ammunition. 2009.

[7] Charles E Loeffler. Detecting gunshots using wearable accelerometers. PloS one, 9(9):e106664, 2014.

[8] David Welsh and Nirmalya Roy. Smartphone-based mobile gunshot detection. In Pervasive Computing and Communications Workshops (PerCom Workshops), 2017 IEEE International Conference on, pages 244-249. IEEE, 2017.

[9] Kenneth L Evans. Effects of recoil on rifle marksmanship simulator performance. Technical report, ARMY RESEARCH INST FOR THE BEHAVIORAL AND SOCIAL SCIENCES ALEXANDRIA VA, 1989.

[10] Double-Alpha Academy. Shotmaxx User Manual. Double-Alpha Academy, Elzenweg 33b, Waalwijk Netherlands, 1.2 edition, 72014.

[11] Artis Mednis, Girts Strazdins, Reinholds Zviedris, Georgijs Kanonirs, and Leo Selavo. Real time pothole detection using android smartphones with accelerometers. In Distributed Computing in Sensor Systems and Workshops (DCOSS), 2011 International Conference on, pages 1-6. IEEE, 2011.

[12] Li Zhang, Parth H Pathak, Muchen Wu, Yixin Zhao, and Prasant Mohapatra. Accelword: Energy efficient hotword detection through accelerometer. In Proceedings of the 13th Annual International Conference on Mobile Systems, Applications, and Services, pages 301-315. ACM, 2015.

[13] Chih-Chung Chang and Chih-Jen Lin. Libsvm: a library for support vector machines. ACM transactions on intelligent systems and technology (TIST), 2(3):27, 2011.

[14] Ron Kohavi. Scaling up the accuracy of naive-bayes classifiers: A decision-tree hybrid. In $K D D$, volume 96, pages 202-207, 1996.

[15] Andrew McCallum, Kamal Nigam, et al. A comparison of event models for naive bayes text classification. In AAAI-98 workshop on learning for text categorization, volume 752, pages 41-48. Madison, WI, 1998.

[16] Johan AK Suykens and Joos Vandewalle. Least squares support vector machine classifiers. Neural processing letters, 9(3):293-300, 1999. 